\title{
Ultra-thin free-standing single crystalline silicon membranes with strain control
}

\author{
A. Shchepetov, ${ }^{1}$ M. Prunnila, ${ }^{1}$ F. Alzina, ${ }^{2}$ L. Schneider, ${ }^{2}$ J. Cuffe, ${ }^{2, a)}$ H. Jiang, ${ }^{3}$ \\ E. I. Kauppinen, ${ }^{3}$ C. M. Sotomayor Torres, ${ }^{2,4}$ and J. Ahopelto ${ }^{1, b)}$ \\ ${ }^{1}$ VTT Technical Research Centre of Finland, P.O. Box 1000, FI-02044 VTT, Espoo, Finland \\ ${ }^{2}$ Catalan Institute of Nanotechnology, Campus de la UAB, 08193 Bellaterra (Barcelona), Spain \\ ${ }^{3}$ Nanomaterials Group, Department of Applied Physics and Center for New Materials, \\ Aalto University School of Science, P.O. Box 15100, FI-00076 Aalto, Finland \\ ${ }^{4}$ Catalan Institution for Research and Advanced Studies (ICREA), 08010 Barcelona, Spain
}

(Received 28 February 2013; accepted 2 May 2013; published online 17 May 2013)

\begin{abstract}
We report on fabrication and characterization of ultra-thin suspended single crystalline flat silicon membranes with thickness down to $6 \mathrm{~nm}$. We have developed a method to control the strain in the membranes by adding a strain compensating frame on the silicon membrane perimeter to avoid buckling after the release. We show that by changing the properties of the frame the strain of the membrane can be tuned in controlled manner. Consequently, both the mechanical properties and the band structure can be engineered, and the resulting membranes provide a unique laboratory to study low-dimensional electronic, photonic, and phononic phenomena. (C) 2013 AIP Publishing LLC. [http://dx.doi.org/10.1063/1.4807130]
\end{abstract}

Silicon has been the dominant material for microelectronics since early 60s. It can be produced with very high crystalline quality, extreme purity, and well controlled electrical properties. Silicon on insulator (SOI) material has been the cornerstone in the development of state-of-the art transistors, ${ }^{1}$ MEMS devices, ${ }^{2}$ opto-mechanical structures, ${ }^{3}$ photonic crystals, ${ }^{4}$ even lithiated anodes for batteries, ${ }^{5}$ etc. Furthermore, thin free-standing SOI structures are ideal to investigate basic physics phenomena in low dimensional structures, such as thermal properties. ${ }^{6-9}$ The most common way to fabricate supported single crystalline Si membranes is to release the top Si layer of a SOI wafer by etching from the backside through the handle wafer and the buried oxide (BOX) layer. However, to obtain very thin membranes, the process usually requires thermal oxidation to thin down the SOI film, followed by removal of the grown oxide. Thermal processes tend to create compressive stress in the SOI film, ${ }^{10}$ leading to buckling of the membranes after release (see Fig. 1(a)). The buckling has been observed in numerous experimental works [see, e.g., Refs. 11-13], and it can be detrimental for device applications exploiting thin free-standing structures. It also complicates experimental work, especially optical measurements, because the angle of incidence of the laser beam may not be well defined. More importantly, the strain, and, consequently, the elasto-mechanical properties of the membrane are unknown. Strain in a Si film can be adjusted, for example, by growing silicon epitaxially on a SiGe buffer ${ }^{14}$ or by depositing thick SiN layers on top and bottom of the $\mathrm{Si}$ layer. ${ }^{15}$ However, these approaches do not allow the fabrication of bare free-standing $\mathrm{Si}$ membranes with tunable strain.

In this Letter, we report on fabrication and characterization of large area ultra-thin flat suspended single crystalline Si membranes with controlled strain and thickness down to

\footnotetext{
${ }^{a)}$ Present address: MIT, Room 7-034C, 77 Massachusetts Avenue, Cambridge, Massachusetts 02139, USA.

b)E-mail: Jouni.ahopelto@vtt.fi
}

$6 \mathrm{~nm}$. Our method involves insertion of a strain compensating $\mathrm{Si}_{3} \mathrm{~N}_{4}$ frame around the $\mathrm{Si}$ membrane perimeter, as shown in Figs. 1(b) and 1(c). The $\mathrm{Si}_{3} \mathrm{~N}_{4}$ layer has small tensile stress that creates a pulling force that flattens the $\mathrm{Si}$ membrane-similarly as a drumhead is tuned. The magnitude of the strain is related to the strain compensation ratio $R_{c}=w_{c} / w_{m}$, where $w_{m}$ is the width of the bare Si part of the membrane and $w_{c}$ is the width of the area of the released membrane covered with the $\mathrm{Si}_{3} \mathrm{~N}_{4}$ film. We show that this approach not only removes the detrimental buckling but also provides an elegant way to control the strain and, consequently, the strain dependent properties such as the energy band structure and elasto-mechanical properties of the lattice without degrading the crystalline quality.

In the fabrication commercially available bonded $150 \mathrm{~mm}$ (100) SOI wafers are used. First, a $20 \mathrm{~nm}$ thick thermal oxide is grown on the SOI wafer and the SOI film is transferred onto a new handle wafer with the $20 \mathrm{~nm}$ thick oxide acting as a new buried oxide layer. The SOI film is thinned by thermal oxidation and oxide stripping to desired thickness. Then a $280 \mathrm{~nm}$ thick $\mathrm{Si}_{3} \mathrm{~N}_{4}$ film under tensile stress is deposited by low pressure chemical vapor deposition (LPCVD) and patterned by UV-lithography and plasma etching, followed by deep etching from the backside through the wafer to release the membranes. The $\mathrm{Si}_{3} \mathrm{~N}_{4}$ frame around the Si membrane provides a pulling force and prevents buckling of the membrane. This approach allows fabrication of flat free-standing single crystalline membranes with size up to several square millimeters and with thickness down to a few nanometers. The fabricated membranes have a square shape with the edges in $\langle 110\rangle$ crystal direction. The crystalline quality of the membranes was verified by high resolution transmission electron microscopy (HRTEM). The images were taken directly from the supported free-standing membranes without transferring them onto a TEM grid to reveal the true condition of the membranes. The surface profiles were measured by optical profilometry and the strain by Raman spectroscopy. 


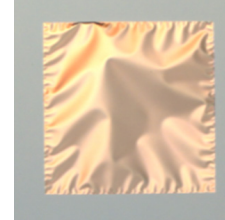

(a)

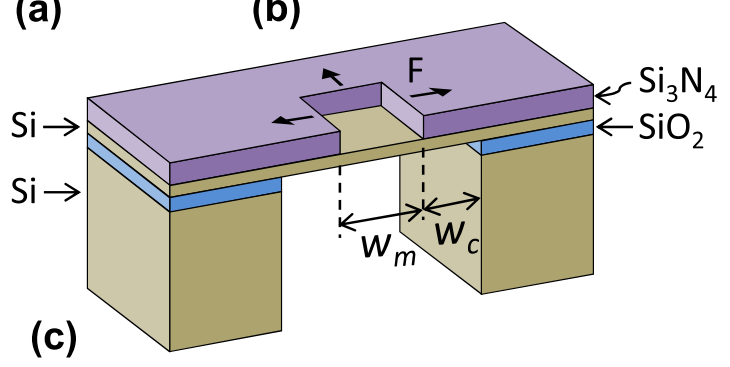

FIG. 1. (a) Optical micrograph of a $37 \mathrm{~nm}$ thick free-standing Si membrane without strain compensation. (b) Optical micrographs of a strain compensated 37 and $6 \mathrm{~nm}$ thick Si membranes. In (a) and (b) the total width of the suspended membrane $w_{s}=1000 \mu \mathrm{m}$ and in (b) $w_{\mathrm{c}}$ is $350 \mu \mathrm{m}$ and $w_{m} 300 \mu \mathrm{m}$, i.e., $R_{c}=1.2$. (c) Schematics of the membrane structure. $w_{\mathrm{c}}$ is the distance from the edge of the membrane to the edge of the stress compensating $\mathrm{Si}_{3} \mathrm{~N}_{4}$ layer and $w_{\mathrm{m}}$ is the width of the bare $\mathrm{Si}$ membrane. $F$ denotes the pulling force due to the $\mathrm{Si}_{3} \mathrm{~N}_{4}$ frame.

The total width of the suspended area $w_{s}$ ranges in these samples from 700 to $1700 \mu \mathrm{m}$. The surface profiles of $37 \mathrm{~nm}$ thick membranes with and without the strain compensation, measured by an optical profilometer, are shown in Fig. 2(a). In both cases the area of the free-standing bare Si membrane is 1000 by $1000 \mu \mathrm{m}^{2}$ and $R_{c}=0.35$ for the compensated membrane. The amplitude of the undulation of the non-compensated membrane is about $4 \mu \mathrm{m}$ whereas the compensated film is completely flat. All the samples with thickness ranging from 6 to $54 \mathrm{~nm}$ analyzed using HRTEM showed no defects and had high crystalline quality, emphasizing that the thickness or the strain does not affect the quality of the membrane. As an example a lattice image of a $9 \mathrm{~nm}$ thick membrane and the corresponding diffraction pattern are shown in Figs. 2(b) and 2(c). The membrane has a strain compensation ratio $R_{c}=1.3$, corresponding to tensile strain of about $1.2 \times 10^{-3}$ as will be shown below.

Raman spectroscopy is a convenient non-destructive and extremely sensitive method to measure strain in the freestanding membranes. ${ }^{16}$ The shift of the LO phonon $\Delta \omega_{L O}$ peak at $\omega_{L O}=520 \mathrm{~cm}^{-1}$ wavenumber reflects the strain in the membrane. Assuming biaxial isotropic stress, the shift of the peak can be translated into in-plane strain $\epsilon_{\|}$as ${ }^{17}$

$$
\Delta \omega_{L O}=\frac{1}{\omega_{0}}\left[q-p \frac{c_{12}}{c_{11}}\right] \epsilon_{\|} .
$$

Here $c_{j j}$ are the stiffness constants, which for $\mathrm{Si}$ are $c_{11}=1.66 \times 10^{11}$ and $c_{12}=0.64 \times 10^{11} .{ }^{18}$ The parameters $p$ and $q$ have values $p=-1.49 \omega_{0}^{2}$ and $q=-1.97 \omega_{0}^{2} .{ }^{19}$

Sets of 6, 27, and $54 \mathrm{~nm}$ thick membranes with different compensation ratio $R_{c}$ were characterized by Raman spectroscopy. The measurements were carried out in ambient at room temperature with excitation at $514.5 \mathrm{~nm}$ at different power levels to exclude the effects of potential heating of the membrane. The beam was focused to a $\sim 1 \mu \mathrm{m}$ diameter spot

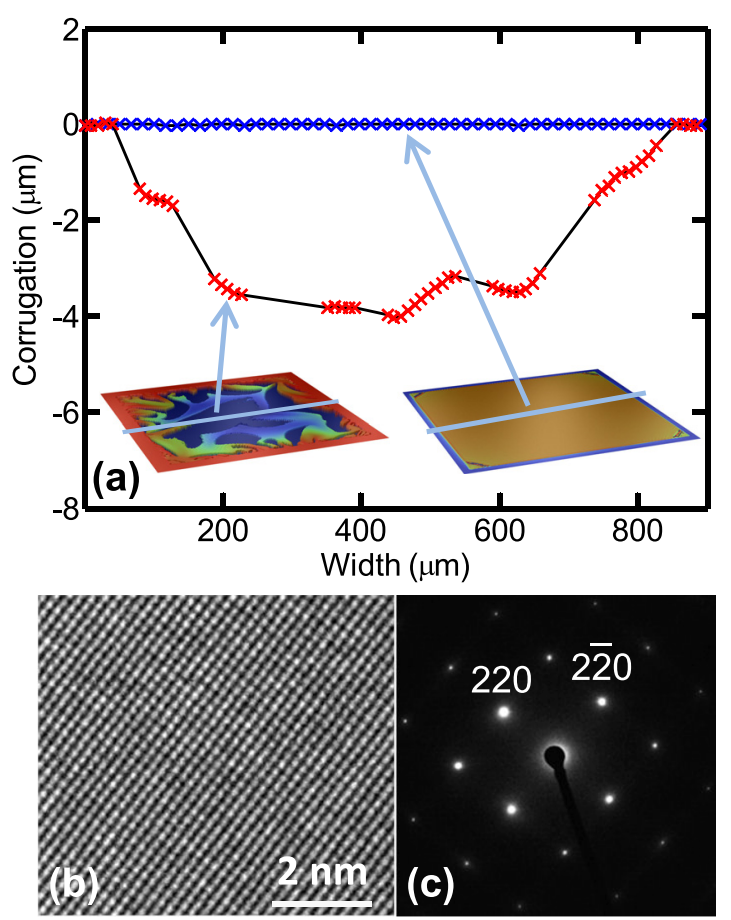

FIG. 2. (a) Surface profiles of $37 \mathrm{~nm}$ thick free-standing membranes measured with optical profilometer. Blue symbols: Surface profile of straincompensated membrane with $w_{s}=1700 \mu \mathrm{m}$ and $w_{m} 1000 \mu \mathrm{m}$, i.e., $R_{c}=0.35$. Red symbols: Non-compensated membrane with $w_{s}=w_{m}=1000 \mu \mathrm{m}$. The missing points result from a too large incident angle for detection. The solid line is a guide to the eye. In the insets lines show where the 3-dimensional profilometer images of the membranes were taken from, (left) without strain compensation and (right) strain compensated. (b) High resolution TEM image of a $9 \mathrm{~nm}$ thick Si membrane with $w_{\mathrm{c}}=400 \mu \mathrm{m}$ and $w_{\mathrm{m}}=300 \mu \mathrm{m}$, i.e., the strain compensation ratio $R_{c}=1.3$. (c) Diffraction pattern of the same membrane.

using a $50 \times$ objective lens. To illustrate the effect of the compensation ratio $R_{c}$ and the thickness of the membrane on the induced strain, LO phonon peaks measured from sets of 54 and $6 \mathrm{~nm}$ thick membranes with various $R_{c}$ of $0.8,1.2$, and 3.5 , together with a reference Si LO peak, are shown in Fig. 3. The shift of the LO phonon peak to lower energy with increasing compensation ratio indicates increasing tensile strain in the membranes. This is the strain tuning effect due to the $\mathrm{Si}_{3} \mathrm{~N}_{4}$ frame, which can be represented in more quantitative way by transferring the peak shift into strain using Eq. (1). Fig. 4 shows the strain obtained from the measured LO phonon peak shifts for the 6,27 , and $54 \mathrm{~nm}$ thick membranes as a function of the compensation ratio $R_{c}$. The behavior is similar for all membranes: The strain increases rapidly for small $R_{c}$ and then begins to saturate at larger $R_{c}$, showing that thicker membranes need more force to build up the strain.

The strain has an effect on many properties of the membranes, such as the mechanical resonance frequencies, Q-value, and electronic band structure. Silicon has six equivalent conduction band energy minima, and the biaxial strain lifts the degeneracy of these valleys. The energy of the two out-of-plane valleys decreases compared to the four in-plane valleys, as shown in the inset to Fig. 4, leading to changes in their relative population. The energy splitting $\Delta E_{c}$ between the in-plane and out-of-plane valleys of the silicon conduction band can be written as ${ }^{20}$ 


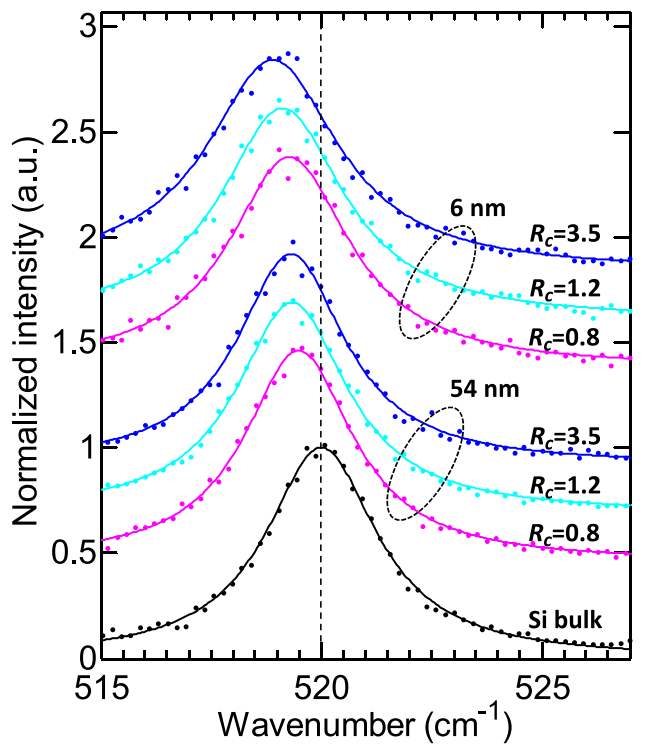

FIG. 3. LO peaks measured by Raman scattering from 6 and $54 \mathrm{~nm}$ thick free-standing $\mathrm{Si}$ membranes with the strain compensation ratio $R_{c}$ of 0.8 , 1.2, and 3.5. The bottom curve is a reference spectrum measured from the bulk part of the Si wafer, defining the reference peak position $\omega_{L O}=\omega_{0}$ in Eq. (1). Solid lines correspond to best fits with Lorentzian function.

$$
\Delta E_{c}=\Xi_{u}\left[1+2 \frac{c_{12}}{c_{11}}\right] \varepsilon_{\|},
$$

where $\Xi_{u}=9.0 \mathrm{eV}$ is the uniaxial deformation potential. ${ }^{18}$ Here, as in Eq. (1), we have used biaxial isotropic stress and zero stress boundary conditions for the bottom and top surfaces of the membrane. The strain induced energy splitting is shown in Fig. 4 in the right axis. At large $R_{c}$ the splitting is of the order of the thermal energy at room temperature. This splitting has an effect, for example, on carrier mobility ${ }^{21}$ and on thermal electron-phonon coupling ${ }^{22}$ in the membrane. The strain affects also the valence bands and the band gap $E_{g}$. There is no simple analytical formula for the energy shift of valence bands. Fit to the simulation results of Ref. 23 gives the band gap change $\Delta E_{g} \approx-19.15 \times \varepsilon_{\|} \mathrm{eV}$ for small strain. The change in the band gap as a function of $R_{c}$ is

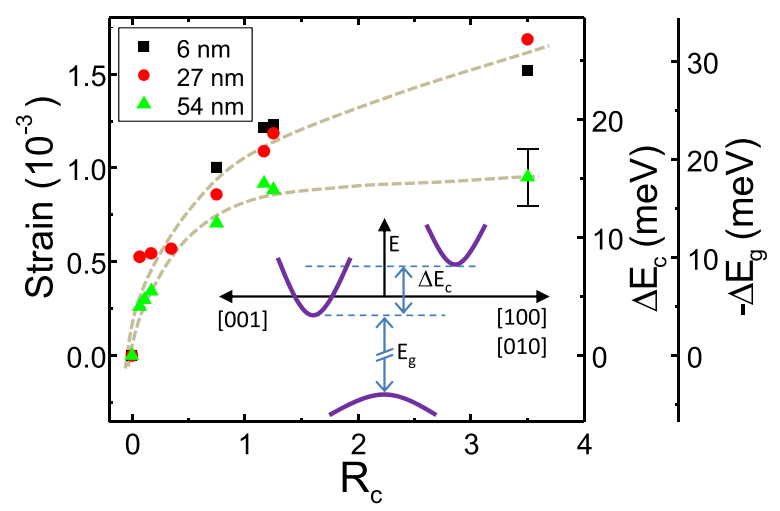

FIG. 4. Strain as a function of strain compensation ratio $R_{c}$ (left y-axis) in $\mathrm{Si}$ membranes with thickness of $6 \mathrm{~nm}, 27 \mathrm{~nm}$, and $54 \mathrm{~nm}$. First right y-axis: Corresponding energy splitting $\Delta E_{c}$ between the in-plane and out-of-plane conduction band minima in Si. Second right y-axis: Change of the band gap energy $\Delta E_{g}$. The splitting of the out-of-plane [001] and in-plane [100]/[010] conduction band minima and the energy band gap are shown schematically in the inset. The dashed curves are guides for the eye, and the error bar is the same for all data points. plotted in the second right y-axis in Fig. 4. The maximum $\Delta E_{g}$ in the current membranes is $\sim 30 \mathrm{meV}$ which can be detected by absorption or photoluminescence measurements.

The LPCVD silicon nitride used in these experiments for strain compensation has relatively low tensile stress of about $300 \mathrm{MPa}$. By changing the growth parameters the stress can be varied between 0 and $1000 \mathrm{MPa}$, providing a mean to increase substantially the strain in the membranes and, consequently, to tune the properties of the membranes. The thinnest membranes fabricated here are $6 \mathrm{~nm}$ thick, corresponding roughly to 20 atomic layers. The membranes are still very robust and preserve their crystallinity surprisingly well, even under the tensile strain. Thermal oxidation is an extremely well controlled process, and the interface between silicon and the thermal oxide is very sharp, of the order of one atomic layer. We expect that free-standing membranes of thickness of 1-2 nm can be realized, having only a few atomic layers remaining. In these membranes reconstruction of the lattice will probably begin to play a role and the theoretically predicted effects in very small crystalline structures $^{24}$ can be investigated experimentally. The role of the native oxide is not clear yet regarding the properties of the thinnest membranes. Native silicon oxide is eventually formed on silicon surfaces in ambient conditions. The thickness of the oxide layer is of the order of $1 \mathrm{~nm}$ but the volume is about twice of the consumed silicon, leading to inhomogeneous thickness of the oxide film and, potentially, local straining of the underlying lattice. Furthermore, the presumed reconstruction of the lattice may alter the physical properties and chemical activity of silicon.

In summary, we report a method to fabricate freestanding Si membranes with thickness ranging down to sub$10 \mathrm{~nm}$ and the strain accurately controlled. The strain enables manipulation of the band structure of Si, permitting engineering of, e.g., electron-phonon and phonon-phonon coupling in a 2-dimensional system, and investigation of the properties of low dimensional systems by optical means due to the largearea of the flat membranes. The fabricated membranes are robust and realization of even thinner membranes with high strain using the demonstrated approach is envisaged.

The authors acknowledge the financial support from the FP7 projects NANOFUNCTION (Grant No. 257375) and NANOPOWER (Grant No. ICT-2010-256959). L.S., J.C., F.A., and C.M.S.T. acknowledge the support of the Spanish projects nanoTHERM (Grant No. CSD2010-0044) and ACPHIN (FIS2009-10150). M.P. and A.S. acknowledge funding from the Academy of Finland (Grant No. 252598).

${ }^{1}$ J.-P. Colinge, Silicon-on-Insulator Technology: Materials to VLSI, 2nd ed. (Kluwer Academic Publishers, Boston/Dordrecht/London, 1997).

${ }^{2}$ J. T. M. van Beek and R. Puers, J. Micromech. Microeng. 22, 013001 (2012).

${ }^{3}$ M. Eichenfield, J. Chan, R. M. Camacho, K. J. Vahala, and O. Painter, Nature (London) 462, 78-82 (2009).

${ }^{4}$ E. Dulkeith, S. J. McNab, and Y. A. Vlasov, Phys. Rev. B 72, 115102 (2005).

${ }^{5}$ C. Yu, X. Li, T. Ma, J. Rong, R. Zhang, J. Shaffer, Y. An, Q. Liu, B. Wei, and H. Jiang, Adv. Energy Mater. 2, 68-73 (2012).

${ }^{6}$ A. Balandin and K. L. Wang, Phys. Rev. B 58, 1544-1549 (1998).

${ }^{7}$ M. Schmotz, P. Bookjans, E. Scheer, and P. Leiderer, Rev. Sci. Instrum. 81, 114903 (2010). 
${ }^{8}$ J. Groenen, F. Poinsotte, A. Zwick, C. M. Sotomayor Torres, M. Prunnila, and J. Ahopelto, Phys. Rev. B 77, 045420 (2008).

${ }^{9}$ J. Cuffe, E. Chavez, A. Shchepetov, P.-O. Chapuis, E. H. El Boudouti, F. Alzina, T. Kehoe, J. Gomis-Bresco, D. Dudek, Y. Pennec, B. DjafariRouhani, M. Prunnila, J. Ahopelto, and C. M. Sotomayor Torres, Nano Lett. 12, 3569-3573 (2012)

${ }^{10}$ T. Iida, T. Itoh, D. Noguchi, and Y. Takano, J. Appl. Phys. 87, 675-681 (2000).

${ }^{11}$ J. A. Rogers, M. G. Lagally, and R. G. Nuzzo, Nature (London) 477, 45-53 (2011)

${ }^{12}$ P. E. Allain, X. Le Roux, F. Parrain, and A. Bosseboeuf, J. Micromech. Microeng. 23, 015014 (2013).

${ }^{13}$ C. M. Sotomayor Torres, A. Zwick, F. Poinsotte, J. Groenen, M. Prunnila, J. Ahopelto, A. Mlayah, and V. Paillard, Phys. Status Solidi C 1, 2609-2612 (2004).

${ }^{14}$ M. M. Roberts, L. J. Klein, D. E. Savage, K. A. Linker, M. Friesen, G. Celler, M. A. Eriksson, and M. G. Lagally, Nat. Mater. 5, 388-393 (2006).
${ }^{15}$ D. Wang, H. Nakashima, J. Morioka, and T. Kitamura, Appl. Phys. Lett. 91, 241918 (2007).

${ }^{16}$ J. Camassel, L. A. Falkovsky, and N. Planes, Phys. Rev. B 63, 035309 (2000).

${ }^{17}$ I. De Wolf, Semicond. Sci. Technol. 11, 139 (1996).

${ }^{18}$ Properties of Crystalline Silicon, edited by R. Hull (INSPEC: The Institution of Electric Engineers, London, 1999).

${ }^{19}$ M. Chandrasekhar, J. B. Renucci, and M. Cardona, Phys. Rev. B 17, 1623-1633 (1978).

${ }^{20}$ C. Herring and E. Vogt, Phys. Rev. 101, 944-961 (1956).

${ }^{21}$ E. Ungersboeck, S. Dhar, G. Karlowatz, V. Sverdlov, H. Kosina, and S. Selberherr, IEEE Trans. Electron Devices 54, 2183-2190 (2007).

${ }^{22}$ J. T. Muhonen, M. J. Prest, M. Prunnila, D. Gunnarsson, V. A. Shah, A. Dobbie, M. Myronov, R. J. H. Morris, T. E. Whall, E. H. C. Parker, and D. R. Leadley, Appl. Phys. Lett. 98, 182103 (2011).

${ }^{23}$ S. Richard, G. Aniel, and F. Fishman, J. Appl. Phys. 94, 1795-1799 (2003).

${ }^{24}$ D. Donadio and G. Galli, Phys. Rev. Lett. 102, 195901 (2009). 